\title{
Endogenous Market Formation and Monetary Trade: an Experiment*
}

\author{
Gabriele Camera \\ Chapman University \\ University of Basel
}

Dror Goldberg

The Open University of Israel

\author{
Avi Weiss \\ Bar-Ilan University \\ Taub Center for Social Policy Research of Israel
}

IZA

August 30, 2016

\begin{abstract}
The theory of money assumes decentralized bilateral exchange and excludes centralized multilateral exchange. However, endogenizing the exchange process is critical for understanding the conditions that support the use of money. We develop a "travelling game" to study the spontaneous emergence of decentralized and centralized exchange, theoretically and experimentally. Players located on separate "islands" can either trade locally, or pay a cost to trade elsewhere, so decentralized and centralized markets can both emerge in equilibrium. The latter maximize trade meetings and are socially efficient; the former minimize trade costs through the use of money. In the laboratory, centralized exchange more frequently emerges when subjects perform diversified economic tasks, but also when they interact in large groups. This shows that to understand the emergence of money it is important to amend the theory of money such that the market structure is endogenized.
\end{abstract}

Keywords: endogenous institutions, macroeconomic experiments, matching, coordination, markets, money.

JEL codes: E4, E5, C9, C92

\footnotetext{
*We thank Avihood Baron, Avichai Chachmon, Inbal Dekel, Isi Feder and Uriel Rochev for research and programming assistance, as well as seminar participants at the Technical University of Berlin and participants at the 2013 and 2015 SAET Meetings and the 2015 ESA International Meetings for comments. D. Goldberg and A. Weiss gratefully acknowledge research support from the Israel Science Foundation (grant No. 1004/11). Correspondence e-mail: camera@chapman.edu
} 


\section{Introduction}

In its most basic form, a model economy is a collection of individuals who exchange the fruit of their specialized labor for something they desire but cannot produce. Consequently, welfare individual and aggregate hinges on the organization of trade. In much of economic theory, trade is assumed to occur in a centralized market characterized by simultaneous multilateral exchange. Yet a sizeable literature, mostly devoted to the study of money and trade frictions, assumes instead that agents trade in a decentralized market characterized by asynchronous bilateral exchanges (Diamond, 1984; Duffie et al., 2005; Lucas, 1984; Kiyotaki and Wright, 1989; Townsend, 1980). ${ }^{1}$

Here we ask: How would individuals choose to organize the process of exchange? This question is important because the organization of trade has implications for allocations and the kind of assets that end up being traded. In particular, we are interested in money. Monetary theorists justify the existence of money in a society as a response to the trade frictions due to decentralized exchange. But the theory of money typically ignores the fact that the mode of market interaction arises endogenously, and simply assumes a decentralized exchange process as a way to capture trade frictions stemming from economic specialization. However, endogenizing the organization of trade is critical for understanding the conditions that lend themselves to the development of money as a mode of exchange. For example, would not specialized individuals want to better organize their exchanges instead of trading pairwise, using money? Theoretical analyses have shown that if agents are free to choose the structure of their market interaction, then equilibria based on decentralized bilateral trade and centralized multilateral trade generally coexist. Decentralized markets are more likely to emerge compared to a centralized marketplace when some object exists that is suitable to serve as a medium

\footnotetext{
${ }^{1}$ The expressions adopted are in line with a long tradition in monetary theory going back to at least Lucas (1984) and Hellwig (1993). "Decentralized market" is typically used to describe an environment in which spatially separated economic agents must travel to a specific location in order to trade asynchronously and in isolation from other economic agents. The contrasting model of exchange is a "centralized market," which is when all agents meet in a specific location and trade simultaneously.
} 
of exchange and when multilateral trade is sufficiently costly (see Camera, 2000; Goldberg, 2007). In this paper we present an experiment designed to provide empirical validation for these theoretical intuitions.

In the experiment, three subjects located on separate "islands" face a trading task that spans multiple rounds of play (a supergame). Subjects are specialized in consumption and in production. Each individual is endowed with one of three differentiated goods in such a manner that everyone derives a benefit from obtaining someone else's good. Hence, there are gains from trade. Due to spatial separation, trade requires coordination on where and when to meet counterparts. In each round, subjects are free to travel round-trip to any one island, sustaining a transportation cost that differs across goods. The problem facing subjects is how to coordinate on a mutually agreeable pattern of exchange in order to maximize their net benefits in the long run. This problem is complicated by the fact that travel choices are made independently, simultaneously and without prior communication.

In this design the basic tradeoff faced by a subject is that his payoff rises with the frequency of consumption but falls with the frequency of travel, the latter particularly if he carries high transportation-cost goods. Economic theory indicates that two basic market structures can emerge in a stationary Markov equilibrium: a decentralized market in which trades are asynchronous and bilateral, and a centralized market in which all trades are simultaneous and multilateral (see Lucas, 1984). In the former, subjects take turns travelling to different islands in order to buy their consumption good while carrying the low transportation-cost good, which thereby serves an explicit medium of exchange function. In the latter, all subjects routinely meet on the same island where endowments are optimally reallocated according to consumption preferences. By design, decentralized trade is less efficient than centralized trade, but the equilibria are Pareto incomparable due to participants' specialization in consumption and production.

We adopt a factorial design. In a baseline condition the environment is composed of a small, stable group: participants are specialized in consumption and 
production because their consumption good and production good are fixed for the entire session, and they also interact for the duration of the session in a fixed group composed of only three trade partners. In subsequent treatments we manipulate specialization, group stability and group size. In the RoTATING treatment participants' consumption and production goods alternate across supergames so that everyone experiences each specialization type. In the REMATCHING treatment groups are randomly re-formed at the start of each supergame, so participants cannot establish long-term relationships during the course of the session. Finally, in the LARGE treatment groups are considerably larger, having between twelve and twenty-four participants so individuals typically interact with different counterparts in each round.

We study three main questions. First, is specialization in consumption and production associated with the spontaneous emergence of decentralized, monetary trade as suggested by the theory of money? Second, does the inability to establish long-term relationships within a stable group of traders also lead to a decentralization of the process of exchange? Third, what is the impact of the group size on the mode of exchange selected by experimental participants?

We find that decentralized markets less frequently emerge when subjects' economic tasks are diversified (Rotating treatment), in line with the theory. Conversely, we do not generally find evidence for the theoretical view that difficulties in establishing long-term relationships have an effect on trade patterns (REMATCHing treatment). Finally, there is evidence that centralized markets more frequently emerge in groups of larger sizes (LARGE treatment); in this case, subjects cannot interact as partners and appear to be more willing to sustain the greater costs associated with setting up a centralized marketplace, instead of trading in isolation from other economic agents by exchanging money. This insight suggests new directions for the study of money, which is typically based on models in which individuals are restricted to meet and trade in pairs, even if they can direct their search (e.g., see Corbae et al., 2003). Our study suggests there is scope to improve the theoretical predictions by incorporating the possibility to 
endogenize the mode of market interaction.

\section{Related experimental literature}

There are several experiments on the use of money in decentralized trading models. Some experiments have studied commodity money systems, as we do, for example the studies in Brown (1996) and Duffy and Ochs (1999). Those studies have primarily implemented designs to test the equilibrium predictions of the commodity-money model described in Kiyotaki and Wright (1989). In that setup, three commodities exist each of which is characterized by a different holding cost. Players start with a production commodity they do not wish to consume and can costlessly barter it for their consumption good through a sequence of bilateral random exchanges. Importantly, players must use a commodity as a money in order to consume because consumption takes place only if someone agrees to trade their production for a good they do not wish to consume. These studies seek to uncover what commodity or commodities will become the medium of exchange and, in particular, whether or not high-storage cost commodities will ever become a "money." In contrast, we study whether or not commodity-money systems spontaneously emerge. In our experiment consumption does not require the existence of a commodity money because players can trade multilaterally and synchronously, and do not need to hold anything but their own production.

Other experiments have studied the endogenous emergence of fiat monetary systems and the behavioral role of money in supporting high-payoff equilibria. In Camera et al. (2013) and Camera and Casari (2014), for example, groups of strangers interact through a random sequence of helping games with unknown opponents. By design, help is non-storable so there can be no commodity monies. However, subjects are endowed with and can exchange intrinsically useless tokens for help, if they wish, thus giving rise to fiat monetary exchange. By design, social efficiency can be attained through a sequence of unilateral transfers of help that do not involve any exchange of tokens. The data show that subjects frequently trade help for tokens, and refuse to help those who cannot offer a token in ex- 
change. That is to say, fiat monetary systems spontaneously emerge even if they are theoretically unnecessary to attain high-payoff equilibria. The use of tokens as a fiat money helps players in coordinating on high-payoff equilibria, especially when groups are large. The related paper in Duffy and Puzzello (2014) adds a centralized market to this basic setup to test the model in Aliprantis et al. (2007). Here, too, fiat monetary systems emerge, although this occurs primarily when groups are sufficiently small. The study of the emergence of fiat money in Huber et. al (2014) takes a different angle by showing that default laws and a "societal" bank can provide a sufficient basis for the use of fiat money. Finally, there is a number of studies in which trade can occur only by using money and all exchanges take place in centralized marketplaces, as in the monetary experiments in McCabe (1989), Marimon and Sunder (1994), Camera et al. (2003) and Deck et. al (2006), for example.

All these studies assume an exogenous organization of exchange. In fact, even if centralized and decentralized markets are available, as in Duffy and Puzzello (2014), subjects are not free to choose where to trade. The novelty of our study is that the organization of exchange is endogenous. Participants in our experiment choose to either synchronize their exchanges in a centralized location, or to engage in a sequence of pairwise asynchronous exchanges in decentralized locations, or to remain in autarky. The two patterns of exchange carry different costs for players, and give rise to two different equilibria: a non-monetary equilibrium based on centralized synchronous trading in which everyone consumes on every trading date, and a commodity-money equilibrium based on decentralized asynchronous trading in which players consume on alternate trading dates. This equilibrium multiplicity gives rise to strategic uncertainty and coordination problems, which vary with the complexity of the task and the size of the group as show in earlier studies, e.g, see Van Huyk et al. (2007), Heinemann et al (1989) and Weber (2006) for example. The experimental literature has focused on pure coordination games in which participants are homogeneous and their incentives are perfectly aligned. By contrast, in our design incentives are misaligned because players are heterogeneous, due to 
their different specializations in production and consumption. Hence, though the equilibrium with synchronous multilateral exchange maximizes social efficiency, neither equilibrium maximizes the payoff of every type of player.

\section{Experimental design}

We start by presenting the BASELINE design, and then discuss four treatment manipulations: Baseline-Rotating, Rematching, Rematching-Rotating, and LARGE (see Table 1).

The travelling game: The BASELINE experiment consists of a coordination task, called the travelling game illustrated in Figure 1. It is played by a group of 3 subjects who can earn benefits by meeting and trading with others.

At the start of the game each subject is randomly assigned to one of three virtual islands $j=1,2,3$. The island $j$ corresponds to the subject's production and consumption specialization type (or simply, type): he produces one good $j+1$ (modulo 3) that is of no value to him, and earns a benefit $u$ only by obtaining one good $j$, which he consumes upon receiving it. To earn this benefit, a subject of type $j$ must meet and trade with a counterpart who holds good $j$. To do so, a subject can stay on his home island, hoping to be visited by other subjects, or can travel round-trip to another island of his choice. Travel is costly; each leg of travel with good $k$ generates a loss $c_{k}$ with $c_{1}<c_{2}<u<c_{3}$. This all but rules out travel with good 3 because travelling to trade good $k$ is individually irrational, as it cannot generate a positive net benefit $u-c_{k}$. We set $u=12$ points, while $c_{1}=1, c_{2}=10$, and $c_{3}=15$ points; 10 points were converted into 1 New Israeli Shekel (NIS) at the end of the experiment. Subjects played multiple rounds of this game. 


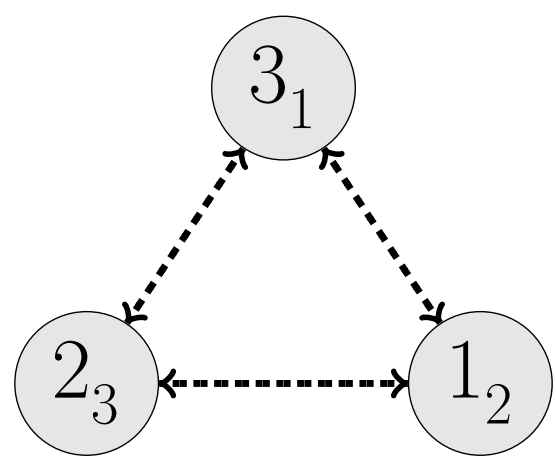

Figure 1: The travelling game

Notes: The larger number denotes the type and island of a player. The smaller number denotes the player's production good. The dotted arrows denote the possible directions of travel. In the experiment type $1=$ fisherman (produces good $2=f i s h$ ), $2=$ farmer (produces good $3=$ bread), and $3=$ hunter (produces good $1=$ fowl).

A round of play: Each round is divided into two stages. In each stage subjects make independent and simultaneous choices, without the possibility to communicate with others.

In the travelling stage subjects observe the distribution of goods across islands and must choose whether or not to travel. As a result of all travelling decisions, a subject can meet one or two other subjects (=bilateral or trilateral match), or can remain unmatched. Matched subjects proceed to the trading stage, in which they participate in a direct mechanism that allows them to re-allocate their goods (=trade). Trade takes place if and only if the matched subjects agree to the proposed reallocation; otherwise, everyone exits the meeting with the good they carried. Hence, there can be either trade or autarky, and unilateral transfers are impossible, which reflects the assumptions of the standard monetary model where all exchanges are quid-pro-quo (Ostroy and Starr, 1990; Starr, 1972).

In a bilateral match, subjects simultaneously choose whether or not to swap their goods; trade occurs if and only if there is mutual consent. In a trilateral match, if all good types are available subjects are given the option to form a trading chain, whereby the subject holding good $j=1,2,3$ transfers it to subject $j$. Trade occurs if and only if there is mutual consent, in which case every subject receives their consumption good. If there is no mutual consent, or if not all good 
types are available in the meeting, then each participant can propose a bilateral trade. Hence, in a trilateral match there are three possible outcomes: there is either one trilateral trade, one bilateral trade, or no trade at all. Round-trip travel implies that each subject $j$ ends the round on island $j$. Subjects who traveled, traded and consumed do not pay any travel cost for the return trip to their island since they no longer have a good in inventory. In the following round, subjects who traded and consumed receive a new good $j+1$, while everyone else carries over their previous round's inventory. Subjects cannot hold more than one good at a time.

A session: Each session involves a multiple of 3 participants $(\min =6, \max =27$, depending on subjects' availability). At the start of the session subjects are divided into groups of three, and each subject is randomly assigned a type $j=1,2,3$. During the entire session subjects interact within the same group and maintain the same type $j$.

Each session consists of several sequences of travelling games. Such a sequence is called a "block;" it starts and terminates simultaneously for all participants. Each block is composed of an uncertain number of rounds of the travelling game. Subjects are informed that they will play six rounds, and from then on, after each round the block will continue with probability 0.75 , and otherwise it will stop. The expected duration of each block is thus 9 rounds. In the experiment, a computer randomly selected an integer number between 1 and 100 from a uniform distribution, and displayed it to subjects. The block terminated when a number greater than 75 was selected. We can interpret the probability 0.75 as the geometric discount factor of a risk-neutral subject (Camera and Casari, 2014). When a block stopped a new one started if there was sufficient time left in the session; otherwise, the session ended. Subjects were informed of this procedure and that they would play at most six blocks.

The design of the block (a fixed even number of rounds plus an additional random number of rounds) allows for the spontaneous emergence of either one of two prototypical trading institutions. First, subjects can resort to centralized trade 
based on one-round trilateral exchange. Second, subjects can choose to engage in decentralized trade based on a two-round sequence of bilateral exchanges. The second type of exchange minimizes transportation costs but requires intertemporal coordination; the first one increases transportation costs but removes the need for intertemporal coordination. Running multiple rounds in a block facilitates learning of a complex task, involving not only travelling decisions but also trading decisions. Because each new block re-initializes the distribution of endowments in a fixed group, running multiple blocks also facilitates learning and coordination on a common trade pattern.

Other treatments: The treatments Baseline-Rotating, Rematching, RematchingRotating, and LARGe, all build upon BASELine.

Recall that in BASELINE subjects repeatedly trade within a fixed group of three participants. In the two REMATCHING treatments trade counterparts change throughout the session: when a block ends, new groups are formed by randomly mixing the session's participants according to a strangers matching protocol.

In BASELINE subjects are specialized in their production and consumption because their type $j$ is fixed throughout the session. In the two Rotating treatments subjects are diversified in their economic activities. Here, the subject's type alternates throughout the session: when a block ends subjects are assigned a different specialization type with round-robin alternation, i.e., 1,2,3,1,2,3; 2,3,1,2,3,1, etc. This design is simply a dynamic interpretation of the static concept of spe-

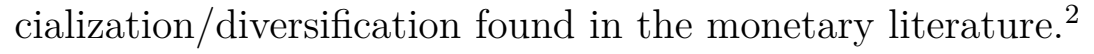

\footnotetext{
${ }^{2}$ In the typical monetary model a specialized agents is one who can produce just one type of good in a period, for their entire life, while a diversified agent can produce one of several types of goods (none of which they can consume).
} 


\begin{tabular}{|c|c|c|c|c|c|c|}
\hline Treatment & Sessions & Size & Groups & Types & Rounds & Blocks \\
\hline BASELINE & $3(30)$ & 3 & fixed & fixed & 55.6 & $6+\mathrm{p}$ \\
\hline BASELINE-R & $3(30)$ & 3 & fixed & mixed & 48 & 6 \\
\hline REMATCHING & $2(42)$ & 3 & mixed & fixed & 42.5 & $4.5+\mathrm{p}$ \\
\hline REMATCHING-R & $2(39)$ & 3 & mixed & mixed & 38 & 5 \\
\hline LARGE: & & & & & & \\
\hline$N=12$ & $2(24)$ & 12 & fixed & fixed & 35 & $4+p$ \\
\hline$N=18$ & $3(54)$ & 18 & fixed & fixed & 34.3 & $3.3+\mathrm{p}$ \\
\hline$N=24$ & $2(48)$ & 24 & fixed & fixed & 28 & $3+\mathrm{p}$ \\
\hline
\end{tabular}

Table 1: Treatments

Notes: $R=$ Rotating. Sessions: the number of sessions conducted (in parentheses the total number of participants). Size: group size. Rounds \& Blocks: average number of rounds played in a session (excluding practice blocks) \& average number of blocks in a session; $+\mathrm{p}=$ session started with an additional, unpaid practice block. Dates of sessions (month-year): BAseline 1-, 3-, 5- \& 6-2014; Baseline-R, 12-2013, 4- \& 5-2014; Rematching, 5- \& 6-2014; RematchingR, 11-2013; LARGE, 3- \& 4-2013, 5- \& 6-2014. In all treatments $u=12, c_{1}=1$, and $c_{2}=10$; $c 3=15$ for all treatments except LARGE where $c 3=20$.

In the LARGE treatment we alter the BASELINE design by increasing the size of groups to $N=12,18,24$, which allows us to study meetings among strangers instead of partners. ${ }^{3}$

Each island $j$ is home to $N / 3$ subjects of type $j$. If $n \leq N / 3$ subjects $j$ stay on island $j$, then the island can host at most $n$ meetings, either bilateral or trilateral, which involve agents of different types. Those who travel to an island are matched at random with those who remained on the island. Hence, an island with $n$ subjects of each type has $n$ trilateral meetings; otherwise, because of the random assignment, trilateral meetings may not occur even if the island hosts all three different types. Finally, if there are more visitors of a single type on island $j$ than subjects of type $j$, then some of the visitors will remain unmatched, though the short side of the market is fully matched. ${ }^{4}$

\footnotetext{
${ }^{3}$ We also have $c_{3}=20$ instead of $c_{3}=15$, but this is both theoretically and empirically inconsequential. LARGE sessions were run first and a small number of subjects accumulated losses by travelling with good 3 (see Table 2). To avoid this, $c_{3}$ was lowered to 15 in all subsequent treatments; doing so is theoretically inconsequential since travelling with good 3 is individually irrational as long as $u-c_{3}<0$, and it did not empirically induce more travel with good 3 (see discussion about travel around Table 2). Therefore, there was neither a theoretical nor an empirical need to rerun all of the LARGE sessions with $c_{3}=15$.

${ }^{4}$ For example, consider a group $\mathrm{N}=12$. If everyone is present on island 3 , then four trilateral matches are formed, each including three different specialization types allocated at random. Now suppose six subjects are on island 3: two type 1, one type 2, and three type 3 . Here, there may be one trilateral match (types $1,2,3$ ) and one bilateral match (types 1,3 ), or there can be
} 
Subject population and aggregate statistics: Overall, we recruited 267 undergraduate students from Bar Ilan University (102 males, 165 females). All subjects were recruited through class announcements, social media and advertisements on campus. We ran 17 sessions (see Table 1 ).

The experiment was programmed and conducted using the software z-Tree (Fischbacher, 2007). Instructions (see Appendix) were read aloud at the start of the experiment and left on the subjects' desks. All sessions were conducted in the Bar-Ilan University experimental lab, where subjects were visually isolated in separate computer carrels. Average earnings per subject were 80 NIS (min $=50 \mathrm{NIS}, \max =122 \mathrm{NIS}) .{ }^{5}$ Payments were structured so that subjects could potentially earn similar amounts per round of play in each treatment. In the Rotating treatments, this was done by summing up all points earned in the session; in the other treatments payments were re-scaled by the maximum points a subject could have earned in the session, given his fixed type (see instructions in Appendix). Subjects completed between 34 and 65 rounds in a session. All sessions lasted two hours including the reading of instructions, a quiz, and payments.

\section{Theoretical considerations}

Time is indexed $t=1,2 \ldots$ There is a constant population of three players who live on three different islands. A player is labeled $j=1,2,3$, which denotes the consumption good of the player as well as the player's home island. Each player $j$ derives utility $u>0$ from consuming good $j$, and discounts future utility at rate $\beta \in(0,1)$. On the initial period, player $j$ is endowed with good type $j+1(\bmod 3)$, which generates no utility to him. Goods are indivisible, cannot be disposed of, and can be stored only one at a time, but can also be traded one-for-one. If player $j$ trades his inventory for good $j$ in period $t$, then he immediately consumes it and is endowed with one new good $j+1$ at the start of period $t+1$. It follows that

\footnotetext{
three bilateral matches (two have types 1,3 and one has types 2,3). If, instead, we had three type 1 , two type 2 , and one type 3 , then there would be only one trilateral match (types $1,2,3$ ) choosing each type 1 with probability $1 / 3$ and each type 2 with probability $1 / 2$.

${ }^{5}$ When the sessions were run the minimum wage in Israel was 24 NIS, while average student wages were around 30 NIS.
} 
all players have some good at the start of each period (see Goldberg, 2007, for a related model with a continuum of players).

Player $j$ starts each period on island $j$ and has the option of making one roundtrip to any island $i \neq j$, during the period. Hence, $j$ denotes player $j$ 's island at the start and end of a period. Carrying good $k=1,2,3$ costs $c_{k}$ for each leg of travel, with $0<c_{1}<c_{2}<u<c_{3}$, so only travel with goods 1 or 2 may benefit a player. Players make their travel choices independently, simultaneously, and without communicating with one another. Hence, a player can either be unmatched, in a bilateral match, or in a trilateral match in any given period. Matched players observe the type and inventory of everyone in their match, and are then asked whether they would like to trade. All trade decisions are made simultaneously without communication with others. Players cannot make unilateral transfers, or give gifts to another player. ${ }^{6}$ In a bilateral match in which the players hold different goods, the player is offered to trade quid-pro-quo with his counterpart. In a trilateral match in which all three goods are available the player is first offered to implement a trading chain. The trading chain is governed by a direct mechanism according to which player $j$ agrees to give a good to one of the two other players conditional on receiving a good from the third player. The proposed exchange is implemented if and only if there is consensus in the match. If one or more players does not acquiesce, the host (the person associated with the island where players are located) is then offered the possibility of a bilateral trade with a player who holds a different goods than he holds. If both other players hold goods that he does not hold, he is asked with which player (if any) he wishes to trade. Bilateral exchange occurs if and only if there is consent from both the party and the counterpart.

There are generally many equilibria in this game, in which actions may or may not depend on histories of play. We focus on Markov-perfect equilibria because the literature on decentralized trade and the microfoundations of money has traditionally restricted attention to these types of outcomes (e.g., see Kiyotaki and Wright,

\footnotetext{
${ }^{6}$ Hence, choosing not to travel and not to trade for the duration of the game is equivalent to free disposal of goods.
} 
1989). In particular, we direct our attention to two patterns of trade that are the focus of theoretical analyses (Camera, 2000; Goldberg, 2007). The first pattern consists of a decentralized trading arrangement in which players trade sequentially and bilaterally on different islands. This dynamic trading arrangement minimizes transportation costs, but reduces the frequency of consumption. The second pattern consists of a centralized trading arrangement in which all players congregate on the same island in each period. This static trading arrangement maximizes the frequency of consumption but increases overall transportation costs.

Figures 2-3 illustrate the two arrangements. The assumption $u<c_{3}$ all but rules out travel of a player 2 holding his production good, under either trade pattern. ${ }^{7}$ Under decentralized trade, two players travel with the lowest transportation-cost good (good 1) on alternate dates and to different islands; on each island there is a bilateral meeting where the visitor exchanges good 1 for his consumption good. Under centralized exchange, the player with the highest transportation-cost good remains on his island, which the other two players visit in each period; on the island there is a trilateral meeting each period in which each player $j$ receives his consumption good from player $j-1(\bmod 3)$ and transfers his good to player $j+1(\bmod 3)$.

\subsection{Decentralized asynchronous trade}

Definition 1 (Decentralized trade strategy). In any period $t \geq 1$ player 1 never travels, while player $j=2,3$ travels only to trade good 1 for good $j$ on island $j-1(\bmod 3)$. If player $j=1,2,3$ is in a bilateral match, then he trades his inventory quid-pro-quo for goods $j$ and 1 . If player $j=1,2,3$ is in a trilateral match, then he transfers his inventory to player $j+1$ only conditionally on receiving good $j$ from someone else.

If all players adopt the strategy in Definition 1, then we say that a decentralized market (DM) emerges in which the asynchronous bilateral exchanges create an explicit need for a medium of exchange. Hence, DM trades support monetary trade. Good 1 serves the role of money because it is not always acquired to be

\footnotetext{
${ }^{7}$ In any Markov equilibrium in which player 2 travels with his production good in some round, he loses even if he consumes in that round. Hence, he can profitably deviate by avoiding travel in that round.
} 
consumed, but, also to be re-traded for another good. Our design thus makes the transactions role of money explicit. Figure 2 illustrates the distribution of objects and the pattern of DM trade in odd and even periods. In equilibrium, player 2 acts as a market-maker who bilaterally trades in every period with alternating counterparts. Trilateral matches never occur in equilibrium, although the strategy specifies what to do in case such meetings occur, off-equilibrium.

In each period the actions of a player depend on his state (his inventory at the start of the period) and the distribution of goods in the economy. The distribution of objects at the start of a period $t$ is such that: player 3 has good 1; player 1 has good 2; player 2 has good 3 in $t$ odd and good 1 in $t$ even. All DM exchanges are bilateral and players trade either to acquire their consumption good or a good that is cheaper to carry than the one they have. Player 1 never moves from island 1 and only one of the other two players the one who has good 1 travels in each period. In odd periods $t=1,3, \ldots$ player 3 has good 1 , and travels to island 2 to buy good 3 from player 2 . In even periods $t=2,4 \ldots$ player 2 has good 1 and travels to island 1 to buy good 2 from player 1 . Off equilibrium, if player $j$ meets more than one counterpart, then he attempts a trilateral trade; otherwise, he attempts a bilateral trade for his consumption good, if possible, or for good 1. If the other two players have an identical good and player $j$ wishes to acquire it, then $j$ trades with the player who consumes his production good, i.e., player $j+1$. 
Travel in $t=1,3, \ldots$

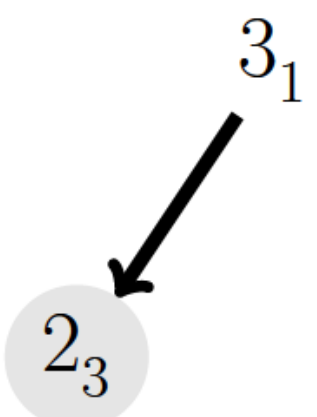

Travel in $t=2,4, \ldots$

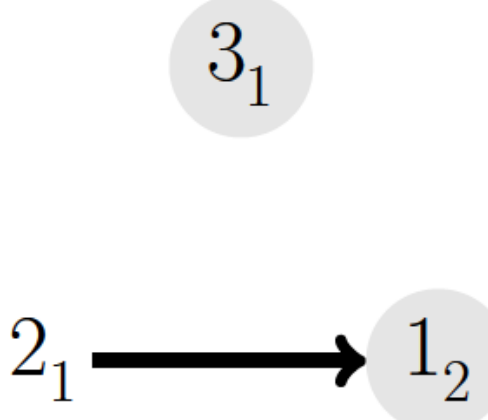

Figure 2: Asynchronous decentralized trade (DM).

Notes: Players in a circle do not travel. The large number denotes the type and island of a player. The small number denotes the player's inventory at the start of the period. The arrow represents the initial direction of travel. In odd periods player 3 travels to island 2 and the two players exchange their inventories. In even periods player 2 travels to island 1 and the two players exchange their inventories. Player 1 never moves. In the experiment type $1=$ fisherman (produces good $2=f i s h$ ), type $2=$ farmer (produces good $3=$ bread), and type $3=$ hunter (produces good $1=$ fowl).

Let $v_{j}$ denote the equilibrium (expected) payoff for player $j$ at the start of a period $s=0,1$, where 1 means odd, and 0 even. When no confusion arises, we omit the distribution of objects and the period $s$ as an argument of $v_{j}$.

Proposition 1. If

$$
c_{1}<u<c_{2}(1+\beta),
$$

then decentralized trade is an equilibrium. The equilibrium payoffs in a period $s=0,1$ are:

$$
\begin{array}{ll}
v_{1} & =\frac{\beta^{s} u}{1-\beta^{2}} \\
v_{2} & =\frac{\beta^{s}\left(u-c_{1}\right)}{1-\beta^{2}} \\
v_{3} & =\frac{\beta^{1-s}\left(u-c_{1}\right)}{1-\beta^{2}},
\end{array}
$$

and average welfare is

$$
v:=\frac{u-c_{1}}{3(1-\beta)}+\frac{\beta^{1-s} u}{3\left(1-\beta^{2}\right)}
$$

The proof is in the Appendix. We need $u \leq c_{2}(1+\beta)$ because if not player 1 would have an incentive to deviate from equilibrium and visit island 2 in odd 
periods. We need $u>c_{1}$ since otherwise player 2 would have no incentive to travel and trade on island 1 in even periods.

To understand welfare, notice that in each period the player who travels (type 3 or 2) also consumes; this gives us the first term in $v$. The other term in $v$ is the consumption of the type 1 player, who never travels, and consumes only in even periods. Note also that given the experimental parameters reported in Table 1 and $\beta=0.75$ we have

$$
v_{2}<v_{1}<v_{3} \text { in odd periods, and } \quad v_{3}<v_{2}<v_{1} \text { in even periods. }
$$

In a decentralized trade equilibrium player 2 acts as an intermediary. He alternates between being a market maker, who makes a market for good 1 (which he cannot consume), and travelling to buy his consumption good. Because travelling is costly and the future is discounted, player 2 is never the top earner. The top earners either do not travel, or trade only for their consumption good.

\subsection{Centralized synchronous trade}

Definition 2 (Centralized trade strategy). In any period $t=1,2 \ldots$ player 2 remains on island 2, while player $j=1,3$ travels to island 2 if and only if every player $j=1,2,3$ holds their production good $j+1$. In every meeting, player $j$ transfers good $j+1$ to player $j+1$ conditionally on receiving good $j$ from someone else. Otherwise, player $j$ keeps his good.

The centralized trade strategy is time-invariant and depends on the distribution of goods across players. In equilibrium, everyone holds their production good and a centralized exchange opens on island 2 in every period. The good that is most costly to transport never moves because players 3 and 1 travel to island 2, while player 2 never travels. Hence, in equilibrium, matches are trilateral and all players synchronously trade and consume in each period. Bilateral matches never occur in equilibrium. Figure 3 provides an illustration. 


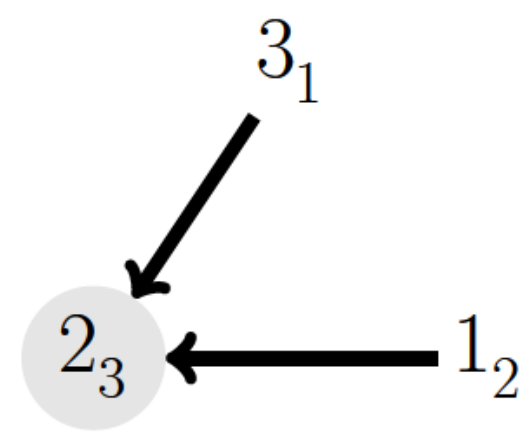

Figure 3: Synchronous centralized trade (CM)

Notes: Players in a circle do not move. The large number denotes the type and island of a player at the start of a period. The small number denotes the player's inventory. The arrow denotes the direction of travel. In every period $t=1,2, \ldots$ players 1 and 3 travel to island 2; player $j=1,2,3$ makes a transfer to player $j+1(\bmod 3)$ conditional on receiving a transfer of good $j$. Player 2 never moves. In the experiment type $1=$ fisherman (produces good $2=f i s h$ ), type $2=$ farmer $($ produces good $3=$ bread), and type $3=$ hunter $($ produces good $1=$ fowl).

If all players adopt the strategy in Definition 2, then we say that a centralized market (CM) emerges. Under this mode of exchange all agents meet in the same location in each period, and concurrently transfer their production to a player and receive their consumption good from a different player. This trade pattern is nonmonetary because all goods that are exchanged are also immediately consumed. This is possible because everyone always carries their production good to the same location, and all exchange is multilateral.

In a $\mathrm{CM}$, player $j$ can deviate by refusing to accept the transfer of player $j-1$ and retaining his inventory. The player can also deviate by not travelling to island 2. Any equilibrium deviation leads to no trade in the period, but does not affect the distribution of goods in the following period. The reason is that trading in a trilateral match is governed by a direct mechanism: if even one player does not receive his desired good, no one makes a transfer. Therefore, every unilateral deviation is suboptimal. This is formally discussed in the Appendix.

Proposition 2. If $u>c_{2}$, then centralized trade is an equilibrium, with payoffs $w_{i}$ to type $i=1,2,3$ given by

$$
w_{1}=\frac{u-c_{2}}{1-\beta}<w_{3}=\frac{u-c_{1}}{1-\beta}<w_{2}=\frac{u}{1-\beta}
$$


and average welfare

$$
w:=\frac{u}{1-\beta}-\frac{c_{1}+c_{2}}{3(1-\beta)}
$$

in each period of the game.

Corollary 1. If $c_{2}<u<c_{2}(1+\beta)$, then equilibria with decentralized markets and centralized markets coexist. Equilibrium with centralized trade maximizes welfare but does not Pareto-dominate equilibrium with decentralized trade.

The proofs are in the Appendix. Equilibrium with CM trade generates more travel costs due to the centralization and the simultaneity of all exchanges. Yet, it also maximizes average welfare because every player consumes in every period, unlike DM trade, and the increment in consumption utility is larger than the increment in travel cost $c_{2}$ relative to DM trade. Neither equilibrium, however, is Pareto-dominant. Type 1 prefers the DM equilibrium because he does not travel; type 2 prefers the CM equilibrium because he consumes every period and bears no travel cost; type 3 prefers the CM equilibrium because he earns $u-c_{1}$ every period instead of every other period.

In the CM equilibrium type 2 also acts as an intermediary, although he no longer makes a market as in the DM equilibrium. Rather, he facilitates trade by maintaining a centralized exchange on his island in every period. Because travelling is costly, type 2 gains from making a market and is the top earner in equilibrium. The lowest earner is now type 1, who has to travel with the highercost good among all goods that are transported. In all treatments both patterns of trade, decentralized and centralized, are equilibria because in the experiment $c_{2}=10<u=12<c_{2}(1+\beta)=17.5$. Therefore, both DM and CM can spontaneously emerge. However, CM trade involves static play as opposed to DM trade, which is based on a two-round cycle of play. This suggests that CM trade might be cognitively simpler for unsophisticated and inexperienced subjects in a laboratory experiment.

\section{Results}

We start with an overview of the data by presenting aggregate information about the incidence of CM and DM play in the experiment. Then, we formulate testable 
hypotheses and present results of the econometric analysis. ${ }^{8}$

\subsection{Aggregate overview}

Table 2 summarizes three distinct pieces of data that help us identify aggregate patterns of trade. For the sake of clarity, we will use the terminology used in the sessions whereby player 1 is a Fisherman who produces fish and consumes fowl, player 2 is a Farmer who produces bread and consumes fish, and player 3 is a Hunter who produces fowl and consumes bread.

Inventory: Consider the top panel in Table 2. Under CM everyone always holds their production good, while under DM Farmers alternate between holding bread and fowl which is the medium of exchange. By design, no one could hold a good they consumed, so if participants of type $j$ did not hold their production good $j+1$ $(\bmod 3)$, then their inventory consisted of the remaining good $j-1(\bmod 3)$. In the experiment, Hunters and Fishermen held their production goods in at least 95\% of the rounds. Farmers held their production good in $74 \%$ of the rounds, which is almost exactly halfway between the two theoretical possibilities of 100 and 50 percent in CM and DM, respectively. This is a first indication that both $\mathrm{CM}$ and DM emerged in the experiment, but neither was predominant.

\footnotetext{
${ }^{8}$ The analysis utilizes blocks 2-6 of each session of the treatments. The first block is excluded because in some sessions it was an unpaid practice block (see Table 1). Those sessions had an additional block 7 , which is also excluded from the analysis.
} 
Table 2: Inventory, travel \& trade decisions

\begin{tabular}{|c|c|c|c|}
\hline Own production & $\begin{array}{c}3=\text { Hunter } \\
\text { fowl }\end{array}$ & $\begin{array}{c}\text { Player type } \\
\text { 1=Fisherman } \\
\text { fish }\end{array}$ & $\begin{array}{c}2=\text { Farmer } \\
\text { bread }\end{array}$ \\
\hline \multicolumn{4}{|l|}{ Theory } \\
\hline CM (Centralized Market) & 1 & 1 & 1 \\
\hline DM (Decentralized Market) & 1 & 1 & .5 \\
\hline \multicolumn{4}{|l|}{ Data } \\
\hline Overall $\mathrm{N}=3034$ & .96 & .98 & .74 \\
\hline Small groups $N=1740$ & .95 & .99 & .72 \\
\hline Large groups $\mathrm{N}=1294$ & .97 & .97 & .77 \\
\hline Travel intensity \& direction & $\begin{array}{c}\text { islands } \\
1,2\end{array}$ & $\begin{array}{c}\text { islands } \\
2,3\end{array}$ & $\begin{array}{c}\text { islands } \\
3,1\end{array}$ \\
\hline \multicolumn{4}{|l|}{ Theory } \\
\hline $\mathrm{CM}$ & 0,1 & 1,0 & 0,0 \\
\hline $\mathrm{DM}$ & $0, .5$ & 0,0 & $0, .5$ \\
\hline All hold production & 0,1 & 0,0 & 0,0 \\
\hline \multicolumn{4}{|l|}{ Data } \\
\hline Overall $\mathrm{N}=3034$ & $.06, .70$ & $.29, .05$ & $.02, .24$ \\
\hline Small groups N=1740 & .05,.65 & $.27, .05$ & $.02, .24$ \\
\hline All hold production $\mathrm{N}=1182$ & $.04, .81$ & $.36, .05$ & $.01, .01$ \\
\hline Large groups $\mathrm{N}=1294$ & $.07, .77$ & $.31, .05$ & $.02, .24$ \\
\hline All hold production $\mathrm{N}=260$ & $.05, .88$ & $.42, .06$ & $.02, .05$ \\
\hline Trade frequency \& modality & Tri, Bi & Tri, Bi & Tri, Bi \\
\hline \multicolumn{4}{|l|}{ Theory } \\
\hline $\mathrm{CM}$ & 1,0 & 1,0 & 1,0 \\
\hline DM & $0, .5$ & $0, .5$ & 0,1 \\
\hline All hold production & 0,1 & 0,0 & 0,1 \\
\hline \multicolumn{4}{|l|}{ Data } \\
\hline Overall $\mathrm{N}=3034$ & $.23, .28$ & $.23, .24$ & $.23, .45$ \\
\hline Small groups $N=1740$ & $.22, .29$ & $.22, .24$ & $.22, .48$ \\
\hline All hold production $\mathrm{N}=1182$ & $.33, .38$ & $.33, .03$ & $.33, .37$ \\
\hline Large groups $\mathrm{N}=1294$ & $.24, .27$ & $.24, .23$ & $.24, .41$ \\
\hline All hold production $\mathrm{N}=260$ & $.37, .32$ & $.37, .04$ & $.37, .20$ \\
\hline
\end{tabular}

Notes: Each cell reports the average relative frequency of observations. One observation $=$ one subject-type in a round; blocks $2-6$, treatments. Own production cells identify how often the player held his own production good at the start of a round. Travel intensity $\&$ direction cells identify how often the player traveled and the island visited (1,2 or 3). Trade frequency $\mathcal{E}$ modality cells identify how often the player traded and the modality ( $T r i=$ trilateral trade, $B i=$ trilateral trade). Small groups $=$ three participants. Large groups $=$ more than three participants. All hold production = observations where everyone in the group has their production good at the start of the round; note that in large groups, at least one unit of each good type was available in every round of the data collected.

Travel intensity \& direction: The middle panel in Table 2 reveals that the 
travel patterns associated with CM and DM share an element of theoretical commonality: no one should travel to island 3 and Hunters should never travel to island 1 . The data are broadly consistent with these travel patterns. There was only a small amount of travel between islands 1 and 3 about 5 percent of the rounds and an even smaller frequency of Farmers' travel to island 3. In particular, travel by Farmers with bread remains minimal in all treatments and the greater travel cost in LARGE treatments $\left(c_{3}=20\right.$ vs. $c_{3}=15$ in all other treatments) did not induce Farmers to travel with bread less than in other treatments. These observations are in line with the theory, since travelling with bread is by design individually irrational.

To identify whether $\mathrm{CM}$ or DM was more prevalent, consider travel choices when everyone held their production good, so that both CM and DM are feasible. As seen in the Table, these can be distinguished by the travel choice of Fishermen, who are pivotal to the establishment of either equilibrium, and travel to island 2 only under CM. In the data, they traveled to island 2 between 36 percent (small groups) and 42 percent (large groups) of the rounds, suggesting that they favored DM over CM trade. This preference is in line with the theory since DM generates higher payoffs to Fishermen than CM. In comparison, Hunters, who should travel to island 2 in every round, did so between 81 and 88 percent of the rounds, slightly more than twice the Fishermen's frequency. The travel patterns are a second indication that both CM and DM emerged in the experiment.

Trade frequency \& modality: Consider the bottom panel in Table 2. Under CM everyone participates in a trilateral trade in every round. Under DM trade is only bilateral, with Hunters and Fishermen trading in half the rounds and only Farmers trading in every period. The data show that Hunters and Fishermen did not trade in every round. The overall frequencies of trilateral and bilateral trade are similar for Hunters and Fishermen. Trilateral trade occurred in 23 percent of the rounds, overall (22 and 24 percent, in small and large groups). Instead, we find that DM trade occurred in 35 percent of the rounds, overall (38 and 31 
percent, in small and large groups). ${ }^{9}$ Again, this is an indication that both CM and DM emerged in the experiment, with slightly greater incidence of DM trade.

Overall, this aggregate evidence suggests that efficiency maximization is not the empirically dominant equilibrium selection criterion in this game. CM trade maximizes the frequency of trade and consumption, and social welfare. Yet, subjects did not select CM more frequently than DM. What influenced subjects' choices in the experiment? We formulate three hypotheses that stem from the theory of money and the experimental literature, and test these using the results in the different treatments.

First, note that $\mathrm{CM}$ trade involves a time-invariant repetition of the same choice in each round of play. This is unlike DM trade, which instead requires intertemporal coordination. The repetitive, history-independent nature of trilateral trade makes the CM strategy cognitively simpler than the DM strategy. Cognitive simplicity is especially valuable to subjects who wish to coordinate on equilibrium play in large groups, because coordination is typically more difficult in large than small groups (Camera et al., 2013; Weber, 2006). This yields the following:

Hypothesis 1 (H1): DM trade is less likely to emerge under LARGE than under other treatments.

Second, the foundations of money literature has put forward the notion that the use of media of exchange naturally emerges when economic interactions are characterized by trading frictions in the form of meetings of short duration with random counterparts (Kiyotaki and Wright, 1989), as opposed to long-lasting meetings between partners. In our setup only DM trade involves the use of a medium of exchange. The Rematching treatment breaks down the long-run interaction in a stable group of BASELINE, because groups are destroyed at the end of each

\footnotetext{
${ }^{9}$ This is not directly discernible from the Table. Rather, it is calculated by counting two consecutive rounds consistent with the two-step sequence in Figure 2. That is, we say that DM trade occurs in two consecutive rounds if in the first round the Farmer does not travel and trades his production for good 1, and in the subsequent round the Farmer travels to island 1 and exchanges good 1 for good 2.
} 
block and randomly re-formed at the start of the following block. As a result we formulate:

Hypothesis 2 (H2): DM trade is more likely to emerge under REMATCHING than under other treatments.

Finally, the foundations of money literature has also put forward the notion that specialization naturally leads to the adoption of media of exchange to facilitate trade (e.g., Camera et al., 2003; Kiyotaki and Wright, 1989). In our setup the Rotating treatment exhibits diversification, as subjects alternate between the different production-consumption specialization types from block to block. As a result we have:

Hypothesis 3 (H3): DM trade is less likely to emerge under Rotating than under other treatments.

Our initial analysis will consider data aggregated at the block level, after which we will turn to individual choice data. Table 3 reports linear regressions based on the aggregated block level data, using the same indicators presented in Table 2. Production share is simply the share of group participants holding own production at start of a round. Each of these indicators, production share, travel intensity and trade volume, should increase with the prevalence of CM equilibria. 


\begin{tabular}{|c|c|c|}
\hline Dep. var.=production share & Model 1 & Model 2 \\
\hline Rotation & $\begin{array}{l}0.035^{* * *} \\
(3550)\end{array}$ & $0.037^{* * *}$ \\
\hline Rematching & $\begin{array}{l}-0.014 \\
(-1412)\end{array}$ & $\begin{array}{l}-0.014 \\
(-1431)\end{array}$ \\
\hline Large & & $\begin{array}{l}0.024 \\
(1.152)\end{array}$ \\
\hline constant & $\begin{array}{l}0.881^{* * *} \\
(36.788)\end{array}$ & $\begin{array}{l}0.870^{* * * *} \\
(41.499)\end{array}$ \\
\hline controls & yes & yes \\
\hline $\mathrm{N}$ & 205 & 229 \\
\hline Dep. var.=travel intensity & Model 1 & Model 2 \\
\hline Rotation & $\begin{array}{l}0.101^{* * *} \\
(5.670)\end{array}$ & $\begin{array}{l}0.100^{* * *} \\
(5.588)\end{array}$ \\
\hline Rematching & $0.043^{*}$ & 0.041 \\
\hline Large & & $\begin{array}{l}0.124^{* * *} \\
(4.773)\end{array}$ \\
\hline constant & $\begin{array}{l}0.392^{* * *} \\
(7.055)\end{array}$ & $\begin{array}{l}0.403^{* * *} \\
(8.496)\end{array}$ \\
\hline controls & yes & yes \\
\hline $\mathrm{N}$ & 205 & 229 \\
\hline Dep. var.=trade volume & Model 1 & Model 2 \\
\hline Rotation & $\begin{array}{l}0.101^{*} \\
(1.874)\end{array}$ & $\begin{array}{l}0.100^{*} \\
(1.941)\end{array}$ \\
\hline Rematching & $\begin{array}{l}0.019 \\
(0.438)\end{array}$ & $\begin{array}{l}0.016 \\
(0.383)\end{array}$ \\
\hline Large & & $\begin{array}{l}0.065 \\
(1.107)\end{array}$ \\
\hline Constant & $\begin{array}{l}0.476^{* * *} \\
(3.898)\end{array}$ & $\begin{array}{l}0.482^{* * *} \\
(4.498)\end{array}$ \\
\hline Controls & yes & yes \\
\hline $\mathrm{N}$ & 205 & 229 \\
\hline
\end{tabular}

Table 3: Production share, Trade intensity and Trade volume.

Notes: Generalized linear model regressions. One observation $=$ average in a block. Blocks 2-6. Model 1: groups $N=3$ only; Model 2: all groups. Production share = share of group participants holding own production at start of a round; Travel intensity $=$ share of group participants who travel in a round; Trade volume $=$ share of group participants who trade in a round. Rotation takes value 0 if participants maintained the same specialization type in each block of the session (1, otherwise); Rematching takes value 0 if participants remained in the same group in each block of the session (1, otherwise); Large takes value 0 if participants interacted in a group with $N=3$ subjects (1, otherwise). Controls include factor variables for blocks 3-6, block duration (number of rounds in the block, number of rounds in preceding block), and fraction of males in the group. $t$ statistics in parentheses with robust standard errors adjusted for clustering at the session level. ${ }^{*} p \leq .1,{ }^{* *} p \leq .05,{ }^{* * *} p \leq .01$

The most robust finding is that there is strong evidence that diversification altered aggregate trade patterns as predicted in hypothesis H3. The estimated coefficients on the dummy variable Rotation in Table 3 are generally highly signif- 
icant. Introducing rotation in roles slightly but significantly tilted the composition of the average inventory toward storing own production (top panel). It also supported a highly significant increase in the intensity of travel (middle panel), and in trade volume, although this last effect is only weakly significant (bottom panel).

Group instability, however, does not appear to exert a significant effect on the aggregate patterns of interaction (H2). The estimated coefficient on the dummy variable Rematching is weakly significant only for travel intensity and only in Model 1 (small economies) of Table 3, and the sign is contradictory to the hypothesis.

Finally, there is evidence that trading large groups led to more travel (H1); the estimated coefficient on the dummy Large in the middle panel of Table 3 is positive, large and highly significant. Yet, we have no evidence of an impact on either inventories or trade volume (top and bottom panels).

These results suggest that CM was more frequently adopted under RoTATION than under BASELINE. The jury is out, however, with regards to whether aggregate trade patterns are affected by LARGE and Rematching. Still, it is important to note that travel intensity for which significant effects were found reflects individual choices, while the other two metrics production share and trade volume reflect an outcome that is affected by collective choices and by circumstances, such as random meetings. It would seem that reaching conclusions requires studying disaggregated data and choices at the individual level, to which we now turn.

\subsection{Individual-level analysis}

We report four main results all of which deal with whether or not REMATCHING, LARGE and/or ROTATION affect the incidence of trilateral trade.

Result 1 (The impact of diversification and group size). Centralized markets more frequently emerged with ROTATION and in LARGE economies than in BASELINE. REMATCHING did not significantly alter the patterns of trade.

Evidence is provided in Table 4, which presents the marginal effects from logit regressions, with the dependent variable taking the value one when there is a 
trilateral trade in a round and zero otherwise.

Models 1 and 2 include all observations, independent of whether a trilateral trade was feasible. Model 1 includes only observations from small economies (one subject of each type) and Model 2 includes larger economies also. Trilateral trade is feasible only if all three good types are available in the economy. The variable $\%$ Feasible $C M$ is the percentage of possible trilateral trades in the economy in that period. In small economies this variable can only be 0 , if some good is not present, or 1 if all goods are present. In large economies, this variable can also take intermediate values because there is more than one individual associated with a player type. Models 3 and 4 include only rounds in which all subjects held their production good, so \% Feasible $C M$ is always equal to one and is therefore omitted.

When subjects rotated across types over the course of the session, there was greater coordination on trilateral exchange. The positive coefficient on the Rotation dummy variable is highly significant. The probability of executing a trilateral trade increases by $24 \%-34 \%$ relative to BASELINE. This indicates that subjects more frequently coordinated on setting up a Centralized Market. The Large dummy variable shows that a similar result holds with respect to large economies, with trilateral trade increasing by 19\%-32\% relative to BASELINE. However, the regressions in Table 4 provide no evidence that REmatching alters the trade pattern. Thus, on the outcome level we have strong support for H1 and H3, but little for $\mathrm{H} 2$. 


\begin{tabular}{lllll}
\hline \hline $\begin{array}{l}\text { Dep Var. =1 } \\
\text { if trilateral trade }\end{array}$ & Model 1 & Model 2 & Model 3 & Model 4 \\
\hline Rotation & $0.245^{* * *}$ & $0.240^{* * *}$ & $0.313^{* * *}$ & $0.336^{* * *}$ \\
& $(3.502)$ & $(2.993)$ & $(3.471)$ & $(3.513)$ \\
Rematching & -0.001 & 0.010 & 0.025 & 0.019 \\
& $(-0.008)$ & $(0.199)$ & $(0.259)$ & $(0.202)$ \\
Large & & $0.190^{* *}$ & & $0.316^{* *}$ \\
& & $(2.200)$ & & $(1.998)$ \\
\% Feasible CM & & $0.475^{* * *}$ & & \\
& & $(5.055)$ & & \\
Controls & yes & yes & yes & yes \\
\hline $\mathrm{N}$ & 1740 & 3034 & 1182 & 1442 \\
pseudo $\mathrm{R}^{2}$ & 0.124 & 0.169 & 0.127 & 0.095 \\
\hline
\end{tabular}

Table 4: The incidence of trilateral trade.

Notes: Marginal effects from logit regression. One observation $=$ one round in a block. All treatments, blocks 2-6. Dependent variable $=1$ if Farmer traded trilaterally in the round. Model 1: $N=3$ groups only; Model 2: all group sizes. Model 3: $N=3$ groups only, and only rounds in which every player stores their own production. Model 4: all group sizes but only rounds in which every player stores their own production. Rotation takes value 0 if participants maintained the same specialization type in each block of the session (1, otherwise); Rematching takes value 0 if participants remained in the same group in each block of the session (1, otherwise); Large takes value 0 if participants interacted in a group with $N=3$ subjects (1, otherwise); \% Feasible $C M$ corresponds to the fraction of CM trades that are possible in a round of groups of any size $N$ (it is defined as the minimum number of goods of any type in the round divided by the number $\mathrm{N} / 3$ of each player type); this variable always equals 1 in Model 4 , so it is omitted. Controls include factor variables for blocks 3-6, sex, and a dummy variable taking value 1 in rounds 6 and above. $t$ statistics in parentheses with robust standard errors adjusted for clustering at the session level. ${ }^{*} p \leq .1,{ }^{* *} p \leq .05,{ }^{* * *} p \leq .01$

To further strengthen these conclusions, the next three results study the individual choices of the two pivotal players: Farmers and Fishermen. Fishermen are pivotal because CM emerges only if they choose to travel to meet Farmers (Figure 3). Farmers are pivotal because DM emerges only if they agree to exchange their production with that of Hunters' (Figure 2).

Result 2 (Travel by Fishermen). Fishermen more frequently travelled to meet Farmers under Rotation and under LARGE than under BASELINE.

The supporting evidence is presented in Table 5. Recall that Fishermen never travel under DM, they always travel (to island 2) under CM, and they derive a greater payoff from DM than from CM. We know that if Fishermen travel, they 
go to island 2 (Table 2). We thus ask: given that both types of markets can be created (CM and DM), do Fishermen travel more in some treatments than others?

\begin{tabular}{llll}
\hline \hline Dep Var. =1 if travel & Model 1 & Model 2 & Model 3 \\
\hline Rotation & $0.338^{* * *}$ & $0.351^{* * *}$ & $0.348^{* * *}$ \\
& $(4.976)$ & $(4.701)$ & $(4.959)$ \\
Rematching & -0.019 & -0.040 & -0.030 \\
& $(-0.235)$ & $(-0.506)$ & $(-0.368)$ \\
Large & & $0.208^{*}$ & $0.251^{* *}$ \\
& & $(1.835)$ & $(2.424)$ \\
\% Feasible CM & & $0.436^{* *}$ & \\
& & $(2.460)$ & \\
Controls & yes & yes & yes \\
\hline $\mathrm{N}$ & 1182 & 2476 & 1442 \\
pseudo $\mathrm{R}^{2}$ & 0.109 & 0.093 & 0.098 \\
\hline
\end{tabular}

Table 5: Fisherman's travel choice.

Notes: Marginal effects from logit regression. One observation $=$ one round in a block when all good types are available. All treatments, blocks 2-6. Dependent variable $=1$ if subject held own production at the start of the round. Model 1: $N=3$ groups only; Model 2: all group sizes. Model 3: all group sizes but only rounds in which every players stores their own production. Rotation takes value 0 if participants maintained the same specialization type in each block of the session (1, otherwise); Rematching takes value 0 if participants remained in the same group in each block of the session (1, otherwise); Large takes value 0 if participants interacted in a group with $N=3$ subjects (1, otherwise). \% Feasible $C M$ corresponds to the fraction of CM trades that are possible in a round of groups of any size $N$ (it is defined as the minimum number of goods of any type in the round divided by the number $N / 3$ of each player type); this variable always equals 1 in Model 3, so it is omitted. Controls include factor variables for blocks 3-6, sex, and a dummy variable taking value 1 in rounds 6 and above. $t$ statistics in parentheses with robust standard errors adjusted for clustering at the session level. ${ }^{*} p \leq .1,{ }^{* *} p \leq .05,{ }^{* * *}$ $p \leq .01$

Table 5 reports the marginal effects from logit regressions regarding Fishermen's travel outcome in a round, when all goods are available. In Model 2 this includes all observations for which at least one good of each type was available, while in Model 3 we only consider rounds in which all subjects held their own production good. In keeping with previous results, we observe a significantly greater probability of travel under Rotation and LARGE than under BASELINE, with the former raising the probability of travel by more than $33 \%$ and Large by more than $20 \%$. Here too, Rematching does not seem to affect the Fishermen's travel decisions. The estimated coefficients on the dummy Rematching in Table 5 are 
the right sign, but insignificant.

Result 3 (Bilateral trade by Farmers). Farmers less frequently agree to bilaterally trade their production good for the Hunter's production under RoTATION and LARGE than under BASELINE.

Supporting evidence comes from the regressions in Table 6, which considers the trade outcome for Farmers who hold bread and are in a bilateral meeting with a Hunter with fowl.

In the meeting Farmers are offered the medium of exchange (fowl) by the Hunter. Farmers earn more from CM than from DM trade, hence they may not want to coordinate on DM trade. If so, then we should see that Farmers are less likely to bilaterally trade for something that is not their consumption good.

\begin{tabular}{lrr}
\hline \hline Dep Var. = 1 if trade & Model 1 & Model 2 \\
\hline Rotation & -0.061 & $-0.134^{*}$ \\
& $(-1.098)$ & $(-1.858)$ \\
Rematching & -0.012 & -0.052 \\
& $(-0.284)$ & $(-0.812)$ \\
Large & $-0.354^{* * *}$ \\
& & $(-3.052)$ \\
Controls & yes & yes \\
\hline $\mathrm{N}$ & 565 & 1034 \\
pseudo $\mathrm{R}^{2}$ & 0.088 & 0.098 \\
\hline
\end{tabular}

Table 6: Trade outcome for a Farmer-Hunter meeting.

Notes: Marginal effects from logit regression. One observation $=$ one round in a block when the subject is in a meeting with a Hunter \& both hold the production good (bread). All treatments, blocks 2-6. Model 1: groups $N=3$ only; Model 2: all groups. Dependent variable $=1$ if subject trades. Rotation takes value 0 if participants maintained the same specialization type in each block of the session (1, otherwise); Rematching takes value 0 if participants remained in the same group in each block of the session (1, otherwise); Large takes value 0 if participants interacted in a group with $N=3$ subjects (1, otherwise). Controls include factor variables for blocks 3-6, sex, and a dummy variable taking value 1 in rounds 6 and above. $t$ statistics in parentheses with robust standard errors adjusted for clustering at the session level. ${ }^{*} p \leq .1,{ }^{* *} p \leq .05,{ }^{* * *}$ $p \leq .01$

There is some evidence that, under Rotation, Farmers are less likely to trade their consumption good for a medium of exchange. The Rotation coefficient is negative and significant in Model 2 of Table 6. The coefficient on the Large dummy 
is negative and significant when we pool all data. In addition, we find that Farmers are more likely to hold their own production good under Rotation. This evidence comes from the regression in Table 7 , where the dependent variable takes the value one in rounds in which subjects in a Farmer role held their production good. A greater probability of holding own production suggests a greater disposition towards adopting CM trade.

\begin{tabular}{lll}
\hline \hline Dep Var. = 1 if hold own production & Model 1 & Model 2 \\
\hline Rotation & $0.088^{* * *}$ & $0.087^{* * *}$ \\
& $(3.644)$ & $(4.010)$ \\
Rematching & $-0.072^{* * *}$ & $-0.070^{* *}$ \\
& $(-2.718)$ & $(-2.523)$ \\
Large & & 0.043 \\
& & $(0.689)$ \\
Controls & yes & yes \\
\hline N & 1740 & 3034 \\
pseudo $\mathrm{R}^{2}$ & 0.022 & 0.013 \\
\hline
\end{tabular}

Table 7: Product held by Farmer.

Notes: Marginal effects from logit regressions. One observation = one round in a block. All treatments, blocks 2-6. Dependent variable $=1$ if subject held own production at the start of the round. Model 1: groups $N=3$ only; Model 2: all groups. Rotation takes value 0 if participants maintained the same specialization type in each block of the session (1, otherwise); Rematching takes value 0 if participants remained in the same group in each block of the session (1, otherwise); Large takes value 0 if participants interacted in a group with $N=3$ subjects (1, otherwise). Controls include factor variables for blocks 3-6, sex, and a dummy variable taking value 1 in rounds 6 and above. $t$ statistics in parentheses with robust standard errors adjusted for clustering at the session level. ${ }^{*} p \leq .1,{ }^{* *} p \leq .05,{ }^{* * *} p \leq .01$

Tables 6 and 7 suggest that RoTATION supports a shift from coordinating on DM to coordinating on CM. REMATCHING does not have an effect on the Farmer's desire to bilaterally trade; the estimated coefficients on the dummy Rematching in Table 6 are insignificant. However, it does have an effect on the Farmer's probability of holding their own production good, and the coefficient on the dummy Rematching in Table 7 has a negative and significant value. Overall, this offers mixed evidence regarding the hypothesis that REMATCHING alters the Farmer's trading behavior.

The results above, have an interesting implication for monetary theory. Start by noting that the LARGE treatment increases the size of the group and introduces 
random meetings, relative to the small BASELINE economies. In the terminology of monetary theory, the LARGE treatment introduces trade frictions because exchange is harder to accomplish than in BASELINE.

Monetary theory suggests that in this kind of situation DM trade should become more prevalent. Individuals would try to raise their consumption frequency by accepting a good that they do not consume but can easily carry and later exchange for their consumption (Kiyotaki and Wright, 1989). In short, uncertain trade opportunities provide an incentive to expand the set of goods that individuals are willing to acquire beyond those that satisfy their effective demand. In practice, Results 2-3 collectively give a different view, which we summarize in the last result.

Result 4 (Trade frictions and the use of media of exchange). Greater trade frictions due to a larger economy size and random meetings, discouraged the emergence of a medium of exchange.

Evidence comes from Table 3, Table 5 and Table 6 . Table 3 shows that frictions are associated with a significant increase in travel (dummy Large, middle panel), suggesting a greater reliance on CM compared to the baseline case. Table 5 shows that Fishermen travel more frequently to meet Farmers under LARGE than BASELINE. Table 6 reveals that Farmers react to an increase in trade frictions by less frequently accepting to trade theirs for the Hunter's production.

Taken at face value, this evidence seems to contradict a basic assertion of the theory of money. However, the typical monetary model imposes a bilateral meeting process while excluding the possibility of altering the meeting process, for example, to synchronized trade in a multilateral meeting. In this case, players have no other option but to accept goods that are not consumed but are easy to carry using money that is in order to increase their consumption frequency.

Result 4 suggests that the theory should be expanded to consider endogenous meeting technologies, without constraining interactions to be bilateral. The experimental evidence indicates that the emergence of media of exchange depends to a large extent on whether or not players are able to easily control their consumption patterns by altering the underlying matching process. 


\section{Discussion}

At an aggregate level neither decentralized asynchronous exchange, DM, nor centralized synchronous exchange, CM, prevailed. Why don't we observe a dominant organization of exchange? A possible reason is that neither trade pattern maximizes the payoff of every player type. By design, some player types (Fishermen) have a greater benefit from engaging in DM trading compared to CM trading (see Section 4). It is thus possible that such a misalignment of incentives made coordination on any given trade pattern difficult to attain. An alternative explanation is that CM trade involves a risky investment for Fishermen, while DM trade does not. Synchronous exchange in a centralized market requires an upfront investment for Fishermen in the form of a high transportation cost. This investment is risky because trade can take place only if all players end up being present at the trading location (Figure 3), which is not guaranteed. By contrast, CM and DM are both risky for Hunters, since they travel in each case (albeit with different frequencies). It is thus possible that such risk pushed Fishermen toward DM trade.

Monetary theory has put forward the notion that the use of media of exchange naturally emerges as a response to trade frictions due to barter difficulties (Kiyotaki and Wright, 1989), specialization (Camera et al., 2003), and the inability to maintain long-run relationships (Townsend, 1980).

Result 1 lends partial support to this view. On the one hand, the experiment provides evidence that decentralized bilateral exchange is relatively more frequent when subjects are specialized and not diversified, as happens in BASELINE relative to Rotating. On the other hand, we do not find evidence that group instability has a significant effect on trade patterns. In the REMATCHING treatment subjects cannot maintain long-run relationships over the course of the session, and yet we do not observe a significant difference in the modalities of exchange compared to BASELINE.

Why does diversification affect trade patterns but group instability does not? Diversification in the RoTATION treatment allows participants to take turns in sharing the greater surplus offered by the efficient CM trade pattern. The alter- 
nation over specialization types is especially meaningful when groups are fixed throughout the session, which unlocks reciprocity schemes. In this case, one can view a session as an indefinite sequence of the travelling games in which endowments are redistributed at random points. According to this view, a subject's lower earnings in an early block can be compensated with higher earnings in subsequent blocks. There is support for this view in the data. Fishermen more frequently travel with their production good when their roles regularly rotate (Result 2). Additionally, Farmers more frequently turn down bilateral trades when their roles regularly rotate (Result 3 ) in order to motivate others to coordinate on CM exchange, which is more profitable for Farmers. On the other hand, introducing instability in a group as done in REMATCHING is likely to weaken reciprocity schemes over the course of the session. Indeed, interaction in an unstable group increases coordination problems and may slow down learning, two elements that are especially important to support DM trade, which requires greater dynamic coordination. This is a possible explanation of the finding that trade patterns are not affected in Rematching when compared to BAseline. Another possible reason is that under REMATCHING subjects may have a stronger incentive to signal their desire to play their preferred equilibrium. However, the incentives are not aligned, because Farmers prefer CM, and Fisherman prefer DM. These two effects may cancel each other out, which is why we do not observe any significant differences in outcomes (inventories, travel and trade) when groups are stable as opposed to unstable.

Dynamic coordination problems are also a likely explanation for the prominence of DM trading conventions in small compared to large groups.

The LARGE treatment prevented repeated interaction in a small group of partners. It more than quadrupled the size of the trading group and imposed a random meeting process. Both of these elements raised coordination difficulties. Experimental evidence indicates that interaction within a stable and small group of partners facilitates coordination and the development of social conventions (Camera et al., 2013; Weber, 2006). Result 4 confirms this intuition because large 
groups more frequently coordinated on CM trade, which is based on a cognitively simpler, time-invariant strategy.

Monetary theorists may express surprise at this result. Monetary theory has put forward the notion that the use of media of exchange should naturally emerge as a response to trade frictions due to random matching in large economies (Kiyotaki and Wright, 1989). The idea is that with greater difficulties in trading, individuals will accept goods they do not consume in an attempt to increase their consumption frequency. And yet, we find that when trading groups are larger and participants meet at random, then centralized markets more frequently emerge because Fishermen travelled more frequently (Result 2) and Farmers refused to trade their production for a good they could transport at low cost but did not consume (Result 3). Does this result contrast with theoretical assertions by monetary theorists? The answer is in the negative. The contrast is only apparent once we recognize that in the typical foundations of money model the trade modality is assumed. For example, in the random matching model of money there can only be asynchronous bilateral exchange, according to some exogenously specified random process. In our design, instead, players are free to bypass trade frictions by organizing their market interactions differently, and exploiting the benefits of multilateral, synchronous exchange. In this sense the experiment shows that to understand the emergence of monetary systems it is important to amend the theory of money to endogenize the mode of interaction. 


\section{References}

Aliprantis, C.D., Camera, G. and Puzzello, D. (2007) Contagion equilibria in a monetary model. Econometrica 75(1), 277-282.

Brown, P.M. (1996) Experimental evidence on money as a medium of exchange. Journal of Economic Dynamics and Control 20(4), 583-600.

Camera, G. 2000. Money, search and costly matchmaking. Macroeconomic Dynamics 4, 289-323.

Camera, G. and Casari, M. 2014. The Coordination Value of Monetary Exchange: Experimental Evidence. American Economic Journal: Microeconomics, 6(1), 290-314.

Camera, G., Casari, M., and Bigoni, M. 2013. Money and trust among strangers. Proceedings of the National Academy of Sciences, 110(37), 14889-14893.

Camera, G., Noussair C. and Tucker, S., Rate of return dominance and efficiency in an experimental economy," Economic Theory, 22, pp. 629-660 (2003).

Camera, G., R. Reed and C. Waller (2003). Jack of all trades or master of one? Specialization, trade and money. International Economic Review 44(4), 12751294.

Corbae, D. Temzelides, T. and Wright, R. 2003. Directed matching and monetary exchange. Econometrica, 71 (3), 731-756.

Deck, C. A., McCabe, K. A., and Porter, D. P. 2006. Why stable fiat money hyperinflates: Results from an experimental economy. Journal of Economic Behavior \& Organization, 61(3), 471-486.

Diamond, P. 1984. Money in Search Equilibrium. Econometrica, 52(1), 1-20.

Duffie, D. and Garleanu, N. and Pedersen, L.H, 2005. Over-The-Counter Markets. Econometrica 73, 18151847.

Duffy, J. and Ochs, J. (1999) Emergence of money as a medium of exchange: an experimental study. American Economic Review 89(4), 847-877.

Duffy, J. and Puzzello,D. (2014) Gift exchange versus monetary exchange: experimental evidence. American Economic Review

Lucas, R.E., Jr. (1984). Money in a theory of finance. Carnegie-Rochester Conference Series on Public Policy 21, 9-46

Goldberg, D. 2007. Money with Partially Directed Search, Journal of Monetary Economics 54(4), 979-993.

Heinemann, F., R. Nagel and P. Ockenfels.2009. Measuring Strategic Uncertainty in Coordination Games. Review of Economic Studies 76, 181-221 
Hellwig, Martin F. 1993. The challenge of monetary theory. European Economic Review 37, 215-242.

Huber, J., Shubik, M. and Sunder, S., (2014) Sufficiency of an Outside Bank and a Default Penalty to Support the Value of Fiat Money: Experimental Evidence. Journal of Economic Dynamics and Control 47, 317-337

Kiyotaki, Nobuhiro, and Randall Wright. 1989. On Money as a Medium of Exchange. Journal of Political Economy, 97, 927-954.

bibitem[Lian and Plott, 1998]Plott Lian, Peng and Plott, Charles R. (1998) General equilibrium, markets, macroeconomics, and money in a laboratory experimental environment. Economic Theory, 12 (1), 21-75.

Marimon, R., Sunder, S. (1994). Expectations and learning under alternative monetary regimes: An experimental approach. Economic Theory 4(1), 131-162.

McCabe, K. A. 1989. Fiat money as a store of value in an experimental market. Journal of Economic Behavior \& Organization, 12(2), 215-231.

Ostroy, J.M., Starr, R.M., 1990. The Transactions Role of Money. Handbook of Monetary Economics, vol. 1, Benjamin M. Friedman and Frank H. Hahn, eds., Elsevier Science Publishers, 3-62.

Roth, Alvin E., and Keith Murnighan. 1978. Equilibrium behavior and repeated play of the prisoner's dilemma. Journal of Mathematical Psychology, 17, 189-98

Starr, R. M. 1972. The Structure of Exchange in Barter and Monetary Economies. The Quarterly Journal of Economics, 86(2), 290-302.

Townsend, R., 1980. Models of money with spatially separated agents. In: Kareken, J., Wallace, N. (eds.) Models of monetary economies, 265-303. Minneapolis: Federal Reserve Bank of Minneapolis

Van Huyk, J., Battalio, R. and Beil, R. 1990. Tacit Coordination Games, Strategic Uncertainty, and Coordination Failure. Am. Econ. Rev. 80, 234-248.

Weber, Roberto A. 2006. Managing Growth to Achieve Efficient Coordination in Large Groups. Am. Econ. Rev., 96(1), 114-126. 